\title{
Évolution des représentations envers le français d'enseignants suisses- alémaniques en formation initiale: une question de temps de formation?
}

\section{Jésabel Robin}

Répondant aux besoins précédemment identifiés dans nos recherches, la nouvelle formation initiale en français langue étrangère que propose la Pädagogische Hochschule Bern aux enseignants du primaire alémaniques s'appuie sur les approches socio-anthropologiques. Un dispositif systémique de «didactique de la mobilité» en trois parties (préparation, accompagnement, réinvestissement) articule désormais les expériences de mobilité dans l'espace francophone avec la pratique professionnelle, les besoins langagiers et le travail sur les représentations sociales du français. Les effets de ce dispositif, conçu sur mesure pour le terrain étudié, sont toutefois en demi-teinte et nous amènent à considérer le rôle de la temporalité dans les aspects processuels de remédiation du rapport au français.

\section{Introduction}

Ancrés en didactique des langues et des cultures (DLC), nos travaux sur le rapport au français des étudiants de la Pädagogische Hochschule (PHBern, littéralement: la Haute École Pédagogique de Berne) ont notamment permis de confirmer que de nombreux futurs enseignants alémaniques du primaire présentent des résistances à l'encontre d'un de leurs futurs objets d'enseignement: cette langue dite étrangère dans les plans d'études bernois. Toutefois, les expériences de mobilité dans l'espace francophone imposées par la formation initiale se révèlent être un lieu-pivot potentiel du rapport au français. Ainsi, la formation en français a récemment fait peau neuve. S'appuyant sur les approches socio-anthropologiques, elle propose un dispositif systémique intitulé «didactique de la mobilité» (Robin, 2017a) articulant désormais la mobilité avec les pratiques professionnelles, les besoins langagiers, et le travail sur les représentations sociales. 


\section{Contextualisation en enchâssement: un terrain sous tensions sociolinguistiques}

\section{Contexte macrosocial: Berne, capitale de la Suisse, pays plurilingue}

La Suisse est, selon une expression de Windisch (2007), une mosaïque linguistique extrêmement complexe. Mises à part les rares communes bilingues, son fonctionnement a trait plus à la juxtaposition de communes à fonctionnement monolingue qu'à un véritable fonctionnement plurilingue. Entre langues officielles et langues nationales, plurilinguisme constitutionnel et principe de territorialité, le statut accordé aux langues (Calvet, 1987) induit des rapports de force qui se traduisent par des relations ambiguës (Widmer, 2004), voire conflictuelles, entre les différentes communautés linguistiques ${ }^{1}$ suisses, dont le tristement célèbre mythe du Röstigraben ${ }^{2}$ est l'exemple le plus significatif. Ainsi, bien qu'elle soit à la fois la capitale d'un pays plurilingue et la capitale d'un canton bilingue, Berne est une commune exclusivement germanophone.

\section{Contexte mésosocial: La PHBern}

Situé en ville de Berne, l'Institut für Vorschulstufe und Primarstufe de la Pädagogische Hochschule Bern est l'institut germanophone de formation initiale des enseignants de l'école primaire du canton de Berne ${ }^{3}$. La formation initiale dure trois années et recouvre 180 ECTS $^{4}$; elle est sanctionnée par un diplôme de Bachelor ${ }^{5}$. Les enseignants sont des généralistes et sont formés à enseigner toutes les matières obligatoires du programme scolaire des degrés scolaires $1 \mathrm{H}$ à $8 \mathrm{H}$ (du Kindergarten à la 6.Klasse). Le français y est la première langue étrangère obligatoire (littéralement Fremdsprache dans les curricula) enseignée dès la $5 \mathrm{H}$ (3. Klasse), à hauteur de trois leçons par semaine avec un manuel imposé. Les étudiants reçoivent ainsi la double formation initiale: enseignant généraliste du primaire et enseignant spécialiste de français langue étrangère (FLE). Le FLE est une matière obligatoire de la formation, à hauteur de 5 à 8 ECTS. Les enseignants du primaire sont ainsi tous de potentiels enseignants de FLE, même si leur formation en la matière demeure très brève.

Cette formation en FLE est composée de cours de langue et de didactique. Elle est validée par des travaux orientés vers la future pratique professionnelle: préparations détaillées de leçons suivant le manuel en vigueur, simulation de séquences didactiques, correction de productions d'élèves, etc. L'enseignement du FLE dans une classe alémanique fait également l'objet de l'un des cinq stages pratiques obligatoires de la formation. Enfin, dans le cadre de cette formation, les étudiants sont tenus d'effectuer au cours de leurs trois années de formation un séjour de mobilité de quatre semaines au minimum dans un espace francophone, en Suisse ou à l'étranger. 


\section{De La résistance envers le français}

Nos précédents travaux ont porté sur les attitudes de résistance envers le français des étudiants de la PHBern; nous y avons montré la nécessité d'aborder ce terrain de manière systémique, c'est-à-dire en postulant la complexité (Morin, 2005) et en considérant les dynamiques entre les contextes macro-, méso- et microsociaux ainsi que celles qui se tissent entre acteurs et institutions. Ils ont exploré cette évidence invisible (Carroll, 1987) largement partagée au niveau macrosocial. Les personnes interrogées pour ces travaux n’ont pas hésité à affirmer qu'il était normal de ne pas aimer le français en Suisse alémanique (Robin, 2015a). Avant d'être une langue, qui plus est une langue nationale, le français est une matière scolaire. Son statut de première langue étrangère obligatoire y est d'ailleurs fortement contesté. Pour nombre d'étudiants, le rapport au français se résume finalement à une contrainte institutionnelle. À la peur et aux souffrances qui accompagnent son apprentissage viennent s'ajouter des exigences élevées en termes de compétences à atteindre. Les écarts entre logiques institutionnelles et logiques individuelles sont alors comblés par des pratiques interstitielles (Robin, 2015b) plus ou moins honnêtes.

C'est le propre d'une représentation sociale, stable et évolutive à la fois, que d'être véhiculée entre pairs sans être questionnée (Jodelet, 1989/1997). Néanmoins, nous avons constaté des mises en conformité de l'expérience avec les représentations sociales (Zarate, 1993), confirmant ainsi la vivacité de ces représentations et limitant l'impact potentiel de toute formation institutionnelle. Ainsi, les représentations ne sont pas toujours construites au travers de l'expérience. S'il semble dès lors difficile d'agir sur le noyau central (Abric, 1989/1997) de la représentation sociale, véritable principe organisateur (Doise, 1986), il est en revanche envisageable d'en infléchir certains aspects, ce qui ne va pas sans poser certaines questions d'ordre éthique, comme: «Â quel titre l'école peut-elle prétendre modifier les représentations des élèves et ainsi infléchir les valeurs héritées de la famille?» (Zarate, 1997, p. 9). À la PHBern, le séjour de mobilité se veut être un de ces lieux d'action potentielle pour la remédiation du rapport au français et le travail sur les représentations (Robin, 2015b). Comme nous le soulignions alors:

Pour nos informateurs en ayant fait l'expérience, l'articulation entre expériences de mobilité en milieu francophone et expériences d'enseignement en milieu francophone semble extrêmement féconde. C'est un lieu-pivot au niveau de leurs représentations et, plus largement, il s'agit d'un tournant réflexif au niveau de la construction d'une pratique professionnelle. (p. 413) Comme on peut le constater sur la carte de langue(s) et de mobilité(s) de Mirjam6 dans la Figure 1, le français n'est vécu qu'au travers des institutions scolaires. Ce qu'elle nomme son "parcours français» est sombre, jalonné d'émoticônes retournés, de compétences barrées et de commentaires négatifs: "notes terribles», "prof ennuyeux», etc. Il s'illumine seulement lorsqu'elle représente ses séjours de mobilité en Suisse romande. 


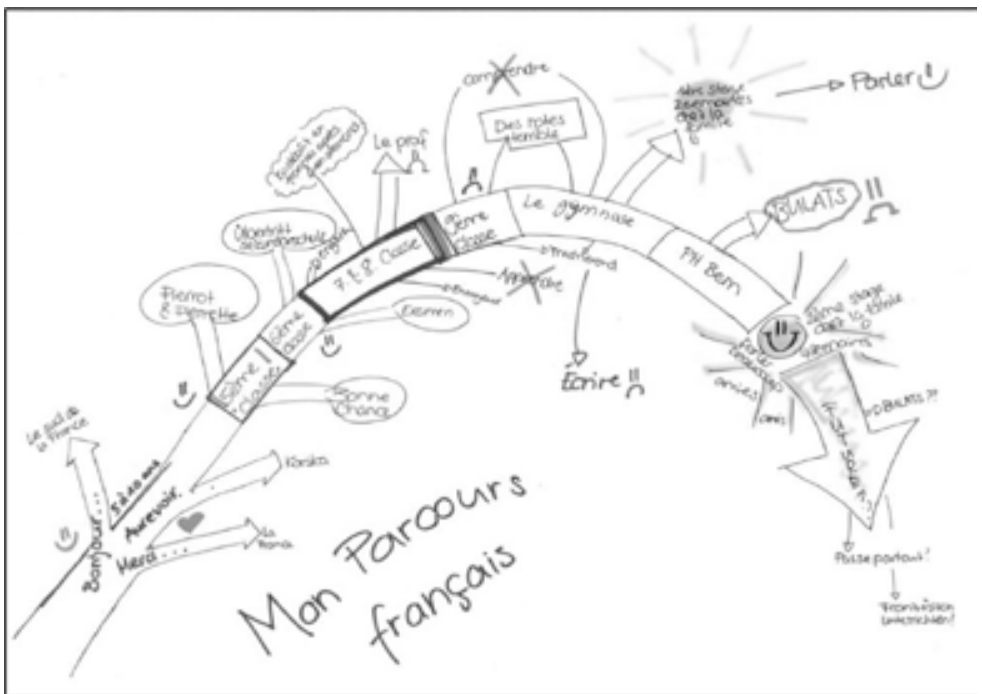

Figure 1: Carte de langue(s) et de mobilité(s) de Mirjam

Par ailleurs, le statut du français en tant que langue de sélection ${ }^{7}$ semble également néfaste aux représentations du français. Son apprentissage a été vécu dans l'angoisse de l'échec par Walter comme on peut le voir dans la Figure 2 ci-après.

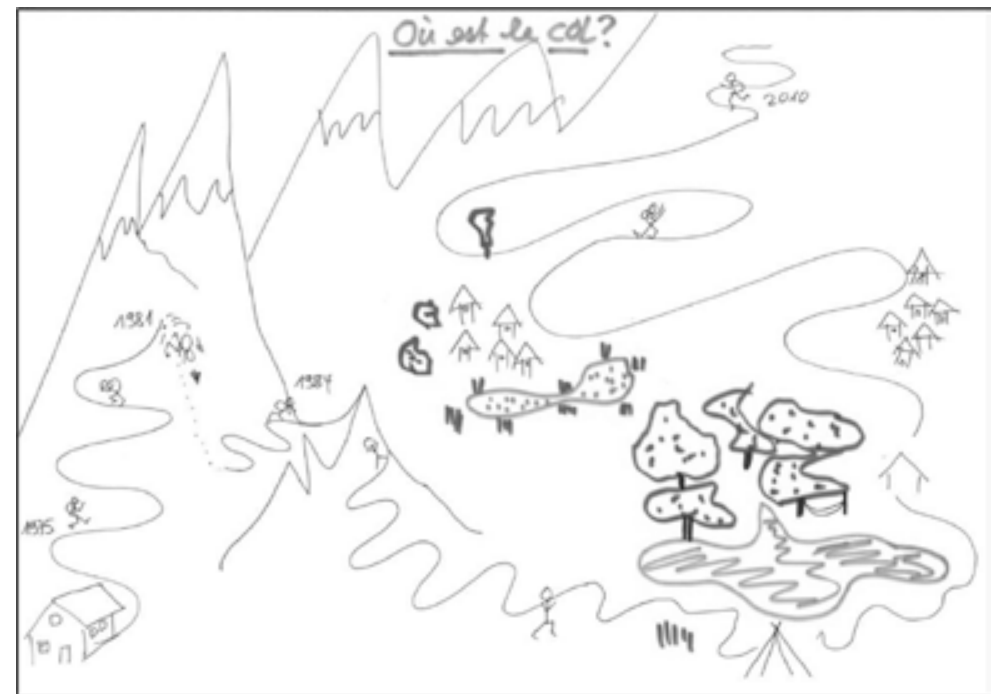

Figure 2: Carte de langue(s) et de mobilité(s) de Walter 
Walter représente son apprentissage du français sous la métaphore de l'ascension d'une haute montagne; ascension périlleuse, de laquelle il chute. Le titre emblématique "Où est le col?» témoignage de la difficulté de son rapport au français. Mais, grâce à son semestre Erasmus en France, le personnage dessiné en recommence l'ascension et semble même pouvoir cette fois gravir le col en courant.

\section{Un nouveau dispositif: Le travail sur les représentations sociales}

Assimilant les expériences de mobilité comme des lieux institutionnels potentiels d'action sur le rapport au français, nous avons conçu un dispositif de «Didactique de la mobilité» sur le principe de la recherche-action-formation ${ }^{8}$. Depuis la rentrée 2016, la contrainte de mobilité en région francophone a fait peau neuve, elle est de plus encadrée. La formation FLE est complétée par un dispositif systémique d'encadrement des mobilités obligatoires en trois parties, étalées sur trois semestres de formation, avant ( $1^{\text {er }}$ semestre) pendant ( $2^{\mathrm{e}}$ semestre) et après $\left(3^{\mathrm{e}}\right.$ semestre).

D'emploi plus récent, le dispositif introduit une souplesse supplémentaire plus proche des réalités rencontrées par les acteurs dans leur pratique. [...] En formation, l'emploi systématique de ce terme depuis les années 1990 met plutôt en valeur l'interrelation entre l'offre et l'usage et la mise en adéquation des formes d'intervention prévues par les concepteurs avec les comportements effectifs des publics destinataires. (Albero, 2010, p. 50)

Le concept de dispositif, dont nous devons l'usage en sociologie à Foucault, considère en effet le dispositif comme un produit social dont la caractéristique serait d'être, comme le décrivent Beuscart et Peerbaye (2006), en perpétuelle reconfiguration pour gérer les effets qu'il produit lui-même, ce qui semble à première vue correspondre aux attentes de la formation initiale de futurs médiateurs de langue(s) et culture(s).

Les dispositifs proposent à l'usager une place à laquelle celui-ci ne peut être indifférent. Leur manière d'inviter à prendre l'initiative valorise certains comportements [...]. Ils attendent de l'usager qu'il s'approprie les ressources mises à sa disposition pour construire un projet personnel. (Peeters \& Charlier, 1999, p. 21)

Il s'agit avec ce dispositif d'éduquer à la mobilité, c'est-à-dire de doter les étudiants d'outils leur permettant de transcender leurs expériences. Cela signifie didactiser, conceptualiser la mobilité pour en faire à la fois un objet d'enseignement et un moyen d'enseignement (par la mobilité). Ainsi, «réfléchir à la manière dont leurs représentations à propos des langues et de l'apprentissage des langues sont empreintes de leurs "cultures d'enseignement-apprentissage» [...] nécessite des compétences de réflexivité qui ne sont pas innées mais qui se 
développent» (Auger, Molinié, Goï \& Guillaumin, 2011, pp. 180-181). Si la préparation linguistique n'est pas oubliée, c'est avant tout un module interculturel qui invite à la décentration, à penser l'impensé comme le dit Laplantine (1987), voire à repenser le déjà pensé (Gohard-Radenkovic, 2009) en vue de la rencontre avec l'altérité. Ainsi l'initiation à l'anthropologie sociale se présente comme «la condition sine qua non pour construire la connaissance de l'autre et de soi» (Gohard-Radenkovic, 2016, p. 22). Dans la plupart des cas, les expériences de mobilité prennent la forme d'un stage pratique supplémentaire, le «stage romand", dans une école de l'espace francophone suisse, articulant ainsi différents types de mobilité: géographique, linguistique, culturelle et professionnelle.

Penser la complexité dans un contexte d'enseignement et d'apprentissage suppose donc de réfléchir de façon systémique, de tisser des liens entre tous les éléments de la situation [...]. J'offre aux enseignants [...] la possibilité d'utiliser des cadres de références leur permettant de penser la complexité. Et qui les distancient du sens commun, de l'intuition. C'est, selon moi, un des rôles principaux que doit remplir la théorie. (Pasquini, 2013, p. 9)

S'appuyant sur les approches socio-anthropologiques en Didactique des langues et des cultures, l'institution n'exige plus de séjour linguistique mais des expériences de mobilité conçues sur mesure pour la PHBern (Robin, 2017b). Ces expériences prennent la forme d'un stage pratique supplémentaire de plusieurs semaines dans une école francophone. Le fait de s'immerger dans un autre système éducatif incite les étudiants à questionner leurs croyances professionnelles et à entrer dans des pratiques de classe différentes de celles dont ils ont l'habitude.

\section{Avant: La préparation aux expériences}

La préparation fait l'objet d'un module obligatoire (3 ECTS des 5 à 8 ECTS) enseigné en français. Convoquant l'interdisciplinarité, il permet au travers de pratiques réflexives de travailler sur ces notions typiques des sciences sociales: représentations sociales, constructions sociales, ethnocentrisme et habitus, en ciblant particulièrement le milieu professionnel.

Que ce soit avec des films documentaires ou bien avec d'authentiques témoignages d'étudiants, les exemples de situations professionnelles à analyser ne manquent pas. Les acteurs de la mobilité conscientisent leurs capitaux en dessinant leur carte de langue(s) et de mobilité(s) ${ }^{9}$.

À titre d'exemple, voici un témoignage original (nous n'avons pas corrigé la langue) que nous utilisons pour décoder les pièges culturels. Il s'agit d'un email reçu d'une étudiante en séjour linguistique en France, relatant une expérience qui l'avait marquée: 
Envoyé: mercredi 4 août

À: Jesabel Robin

Objet: france

Salut Jésabel

J'écris seulement pour te ${ }^{10}$ dire que cette projet de l'assistance de langue m'a mis à L. C'est super, c'est en Bretagne et c'est près de la mer:-) juhuuu!

Bon, je peux bien imaginer que l'hiver ne sera pas si beau. Mais bon, je suis certain que je vais profiter pour mon futur enseignement.

Malheureusement il y avait un truc assez shit. Ecoutes: J'ai contacté une prof en e-mail et elle m'a répondu tout sympha. Parce qu' elle m'a tutoyé et le son et le contenu me semblait assez familier et elle a mis son prénom et son nom, moi aussi j'ai la tutoyé la prochaine fois.

$\mathrm{O}$ là là, mauvaise idée. Son mari m'a répondu pour me dire, que je devrait vouvoyer les profs, c'était une règle assez basale. Point! Oops. Je me suis éxcusé, mais il n'a pas vraiment réagi.

Est-ce que c'est normal?!? Je pense que c'est un peu bizarre, si elle avait écrit d'une façon plus formelle et si elle avait mis que son nom de famille, j'aurait la vouvoyé, c'est clair.

Qu'est-ce qu'ils veulent comme éxcuse encore? Du Chocolat? Fromage? Des Montres?

Quand j'étais la dernière fois en France tout le monde m'a donné deux bisous au lieu de dire bonjour, ça aussi était un peu extrème pour une Suisse:-/ Bof, je vais lui écrire encore une fois d'une manière super poli avec tous les phrases nécessaires..

Voilà mon petit histoire.

S.

En groupe, les étudiants tentent de répondre aux questions suivantes: Quel est le problème? Quelle est la réaction de l'enseignante? La comprenez-vous? Quelle est la réaction de S.? La comprenez-vous? Que faire à présent et comment répondriez-vous à $S$.? L'analyse de la situation permet de mettre au jour les codes implicites du vouvoiement/tutoiement en France et, en miroir, de forcer les étudiants alémaniques à expliciter leurs propres codes (implicites bien entendu mais qu'ils considèrent comme évidents et qui ne méritent donc pas d'être explicités): principe de réciprocité entre adultes, signal du prénom, etc. En déconstruisant les constructions sociales, les étudiants réalisent que les deux acteurs de cette situation ont agi correctement selon les codes de leur groupe respectif. Cela permet de désamorcer un conflit potentiel tout en gardant la face (Goffman, 1973) des deux acteurs. Pour conclure, nous leur présentons le témoignage d'une étudiante wallonne en semestre d'échange à la PHBern et qui s'étonne, elle, des pratiques du tutoiement en Suisse alémanique. 
Ici en Suisse, on met l'accent sur l'égalité entre tous ce qui me déstabilise encore profondément. Lors de mon stage, l'enseignante bien plus âgée que moi, m’a proposé-de la tutoyer et de l'appeler par son prénom. Cela m’a mis mal à l'aise. Je n'ai pas cessé d'osciller entre des 'vous' et des 'tu' ne sachant pas trop que faire de cette nouvelle norme sociale.

Bien que les autres étudiants parlent de ces normes comme des évidences, mettant en avant les valeurs démocratiques de la société dans laquelle ils vivent, je pense que je ne m'y habituerais pas d'aussitôt. A.L.

Nous travaillons ainsi, toujours sur la base de témoignages authentiques d'anciens étudiants en mobilité ou de films documentaires, des sujets tels que la ponctualité, l'autorité, la relation adulte-enfant, les rapports enseignants-élèves, l'espace scolaire ouvert-fermé, l'organisation spatiale et temporelle de l'école, la distance et le contact physique entre les acteurs scolaires, les rituels de classes, etc. en replaçant soigneusement à chaque fois le témoignage et/ou l'évènement relaté dans son contexte social de production afin d'éviter de recréer des stéréotypes et en ayant toujours à cœur de nuancer. Ainsi les étudiants prennent conscience de ce qui est construit chez eux et chez l'autre, le but n'étant pas tant de comprendre pourquoi, mais de comprendre comment fonctionnent les mécanismes sociaux et en particulier dans le milieu professionnel ciblé. Ainsi le travail sur les représentations sociales passe avant tout par un travail sur ce que Carroll (1987) a appelé les prémisses culturelles, c'est-à-dire sur les références premières.

\section{Pendant: L'accompagnement des expériences}

L'accompagnement des expériences a été pensé pour être forum interactif permettant d'échanger entre paires pendant les expériences. Basé sur le concept du journal d'étonnement (Develotte, 2006), les acteurs de la mobilité peuvent s'y étonner ensemble et essayer de comprendre, c'est-à-dire d'objectiver ensemble (comme cela a été pratiqué dans le module de préparation). Le forum est en ce sens un dispositif de co-explicitation avec les acteurs (Vinatier, 2009). Il offre en théorie (nous allons voir qu'en pratique il en est allé tout autrement) la possibilité de suivre l'évolution des représentations in vivo (Dervin, 2007) et constitue à ce titre un moyen potentiel d'observation en temps réel d'éventuels processus de remédiation et donc, indirectement, est en soi un outil d'évaluation des apports du dispositif.

\section{Après: Le réinvestissement des expériences}

"Avoir vécu une expérience ne suffit pas pour que cette expérience devienne de l'expérience. Il faut sans cesse la renégocier et la re-méditer» (Morin, 1959, p. 10). Pour passer de l'ethnocentrisme à la relativité, les formateurs doivent développer un regard anthropologique (Veillette \& Gohard-Radenkovic, 2016). Les sciences sociales fournissent en effet les outils qui permettent de questionner les représentations sociales, les évidences, les discours, le rapport 
à l'autre, de déconstruire les faits culturels observés (Berger, 2006). Elles se retrouvent finalement au cœur des préoccupations de la formation de formateurs et occupent une place importante dans les dispositifs de formation à la mobilité. Seule une approche pluridisciplinaire et systémique permet de prendre en compte la complexité.

À leur retour, les étudiants sont invités à «mettre les expériences de mobilité au service de la formation professionnelle» 11 à l'occasion d'un module obligatoire recouvrant quatre heures en présentiel. Ce module n'est pas forcément donné par des enseignantes de l'équipe de français et tous les échanges - écrits et oraux - peuvent avoir lieu en allemand. Les séances sont avant tout l'occasion de partager et de verbaliser de nouveau, en face à face cette fois. Cela peut également être le moment de revenir sur les attentes envers les expériences exprimées par écrit dans le module de préparation et de voir dans quelle mesure les objectifs individuels de chacun ont été atteints, ou non. Au niveau institutionnel, c'est ce réinvestissement (Reinhardt \& Rosen, 2013a) qui est au cœur des préoccupations. C'est d'ailleurs en se situant par rapport à leurs expériences qu'il est possible de les transformer en capital professionnel (Vinatier, 2009).

Nous exploitons volontiers les contre-exemples qui nous sont soumis. Ainsi, une étudiante osa un jour intervenir (avec une toute petite voix qui semblait signifier: surtout ne vous fachez pas Madame, je ne souhaite pas vous manquer de respect, mais...: «Madame, je crois que vous vous êtes trompée un petit peu lors de la préparation avec cette histoire de vouvoiement... Moi, j'étais dans une école en France, et c'était pas du tout comme vous avez dit, tout le monde se tutoyait». Ce genre d'intervention est une véritable aubaine pour notre travail, qui permet encore une fois de nuancer, voire de rectifier le tir s'il y a eu malentendu. Certains semblent développer une obsession des différences au risque d'occulter les similarités, qui sont avant tout (il faut le souligner) très nombreuses dans le domaine éducatif entre les aires francophones et alémaniques suisses. En effet, il ne s'agit certainement pas de recréer un stéréotype du genre: «les Français vouvoient et les Suisses allemands tutoient» (dixit). En interrogeant davantage l'étudiante sur ses expériences et surtout en contextualisant au maximum, elle a fini par expliquer que dès son premier jour de stage, son enseignante-formatrice l'avait informée que le tutoiement était la norme au sein de l'établissement. Nous avons ainsi pu revenir et approfondir les aspects implicites/ explicites des codes sociaux. En contextualisant les situations de formation et en tenant compte de la complexité mais aussi de la multiplicité des individus et de leurs expériences, on tente alors non pas de figer les différences mais d'appréhender les attitudes face à la diversité et de les dépasser. Ainsi, la reconfiguration des expériences a posteriori fait partie d'un potentiel processus de remédiation du rapport au français et de ses représentations sociales.

En outre, lors des séances d'échanges, les étudiants sont invités à s'exprimer sur le dispositif lui-même afin de le réaménager. Ils agissent ainsi directement en tant que co-acteurs de l'institution et la modélisent, ce qui crée une interaction 
complexe et une dynamique sociale entre les différents acteurs et co-acteurs de l'institution.

\section{Apports et limites du dispositif}

\section{Les apports}

La RAF «Didactique de la mobilité», financée pour trois années (2016-2019) par l'Institut für Forschung, Entwicklung und Evaluation de la PHBern, permet d'évaluer les apports d'un tel dispositif. Nous avons analysé les évolutions potentielles des représentations de cinq étudiants sélectionnés puis suivis de manière longitudinale pendant leurs trois années de formation au moyen d'entretiens compréhensifs, de carte(s) de langues et de mobilités (Robin, 2014), d'une fiche de données et de leur travail de réflexion ${ }^{12}$. Les analyses qualitatives menées au moyen de catégories conceptualisantes (Paillé \& Mucchielli, 2012) montrent à ce stade que, fonctionnant sur l'axe paradigmatique du proche et du loin, c'est bien en se distanciant de leur formation initiale suisse alémanique que les étudiants parviennent le mieux à analyser leur processus de formation: "Le système éducatif de la France [...] m'a aidé d'observer le système éducatif de la Suisse d'une autre et nouvelle façon» (travail de Lisa). Le témoignage de cette étudiante indique par ailleurs que des prédispositions (Murphy-Lejeune \& Zarate, 2003) favorables au développement d'un capital plurilingue et pluriculturel (Zarate, 2012) sont nécessaires pour tirer profit de la rencontre: "Je ne pensais pas que mon semestre d'échange à Toulouse me donnerait envie de voyager le monde arabe. C'est une surprise géniale." (travail de Lisa). Cette étudiante qui déclarait avant son départ que son objectif premier était de "rencontrer des Françaises» 13 (fiche de Lisa) a en effet eu l'opportunité de rencontrer des personnes issues du «monde arabe» et a su laisser cette altérité inattendue l'enrichir personnellement.

Le témoignage de Patricia montre également des évolutions: elle a pris conscience de certaines des constructions sociales liées à ses appartenances premières et à son habitus professionnel en construction. Elle accepte de les déconstruire en situation d'altérité pour réussir un échange professionnel de haute volée avec une enseignante francophone: "J'ai expliqué mon point de vue et ce que j'ai appris et donc pourquoi moi j'interviens comme ça. Comme ça je devais remettre en cause mes propres attitudes et argumenter" (travail de Patricia).

Comme le soulignent les chercheurs, l'un des problèmes majeurs de ce type de dispositif exploratoire est d'assurer sa pérennité au-delà des années du financement de projet (Clerc \& Richerme-Manchet, 2016). Notre dispositif d'encadrement des expériences de mobilité s'inscrit de facto dans la durée puisqu'il est désormais inscrit dans les plans d'études et partie intégrante de la formation initiale des enseignants du primaire. Pour les semestres d'échange académique, quelle qu'en soit la destination, nous étudions actuellement l'éventualité de donner accès aux étudiants de la PHBern au programme facultatif d'enca- 
drement Mentoring Intercultural Learning through Study Abroad (MILSA) ${ }^{14}$ proposé par le bureau des Relations Internationales de l'Université de Berne.

\section{Les Limites}

«Les modules de français sont déjà peu nombreux et répondent à d'autres objectifs et besoins de formation (notamment linguistiques et didactiques), laissant finalement très peu de place à ce fragile dispositif d'accompagnement» (Robin, 2013). Si nous pensons avoir tout de même réussi - malgré le cadre restreint que nous offre l'institution - à didactiser la mobilité dans le contexte précis des expériences de mobilité de la PHBern et avoir ainsi aidé à développer un habitus mobilitaire (Gohard-Radenkovic \& Veillette, 2015) auprès de nombreux étudiants, nous ne sommes pas à l'abri d'entendre ou de lire encore des déclarations culturalistes ou de voir des représentations négatives du français et de ses locuteurs, sinon se renforcer, en tout cas stagner.

La tentation est toujours la même, pour tous les acteurs sociaux, et les enseignants n'échappent pas plus que les autres à ce destin-là: celle de prendre sa position pour la position, c'est-à-dire la seule possible. On est toujours tenté de considérer que le monde est objectivement identique à la manière dont on le voit. (Porcher, 1997, p. 25)

Les réflexions des étudiants se cantonnent souvent au mode binaire de la comparaison "eux versus nous» sur le paradigme du proche et du lointain développé par Simmel (1999). Les dynamiques du rapport à la diversité et la remédiation des représentations sociales ne sont donc pas toujours ascensionnelles, comme l'indique la récurrence du "chez nous» dans le discours de Peter: "C'est plus ou moins le même contenu que chez nous. C'est un peu plus scolaire que chez nous. On joue pas si beaucoup que chez nous» (entretien avec Peter).

Nous savons certes, que les représentations sociales reflètent l'appartenance macrosociale (Zarate, 1993) et nous donnent en l'occurrence à voir autant sur le contexte sociétal des rapports entre communautés linguistiques suisses que sur des intériorités. Tout de même, elles prouvent que les dynamiques sont loin d'être enrayées et nous rappellent la modestie à adopter en tant que concepteur de RAF. Il y a toujours des écarts entre les objectifs formulés au niveau institutionnel et la réalité du terrain. La formation institutionnelle ne peut agir que sur un terrain fertile (prédispositions, habitus favorables, etc.) et la vitalité des représentations sociales limite forcément l'impact de tout dispositif (Robin, 2015a).

La mise en scène de mea culpa orchestrés, de retours pseudos réflexifs où les étudiants sacrifiant à une contrition et à une auto-évaluation superficielles, voire factices, peut devenir une stratégie efficace pour contourner l'injonction à la réflexivité et tenter d'obtenir une «bonne» évaluation de la part des enseignants. (Goï \& Huver, 2011, p. 203)

Si elles seules permettent de respecter la complexité du terrain, les pratiques réflexives, une fois institutionnalisées par la formation, présentent elles aussi 
leurs limites. Ainsi, les potentiels apports de ce nouveau dispositif sont à relativiser dans tous les cas.

En outre les apports des technologies de l'information dans le dispositif ont été un échec total. Le forum n'a pas du tout été utilisé, les acteurs préférant échanger à titre privé «entre collègues» plutôt qu'en public sur un forum, d'autant plus volontiers qu'ils avaient pour ce faire d'autres canaux de communication à disposition.

\section{La temporalité dans la remédiation}

\section{Penser en termes de processus}

Évaluer dispositif ne signifie pas le légitimer par rapport à son éventuelle rentabilité. Le rapport qualité/prix (Chopin, 2010) entre le temps investi dans la formation et le résultat obtenu, c'est-à-dire la question de l'efficacité du temps scolaire (Morlaix, 2006), se pose ici plutôt en termes processuels.

«On est frappé par l'insistance des étudiants sur la valeur existentielle de la période [de mobilité] comme expérience de vie» (Anquetil, 2006, p. 45). Le récit est un instrument à même de faire ressortir les éléments que l'informateur juge pertinents. Il permet de s'étendre largement, et donc de ne pas respecter la chronologie ni les proportions de la durée dans le temps des épisodes jugés comme habituels. Baudouin (2009) nomme "distal» ces moments de rupture avec l'habituel dans le récit. Les expériences de mobilité de nos informateurs fonctionnent comme du distal dans leurs récits.

Appréhendant la pluralité linguistique, culturelle et professionnelle, le nouveau dispositif d'encadrement des expériences de mobilité de la PHBern met au centre des préoccupations le contact avec cette soi-disant "altérité» intra-nationale francophone. Si les changements de conceptions de la mobilité sont la preuve que de nouvelles dynamiques institutionnelles se mettent en place, il est encore trop tôt pour juger de leurs répercussions sur la diversité des expériences individuelles. Toutefois, en pariant sur un dispositif en trois temps (avant/préparation, pendant/accompagnement, après/réinvestissement) et s'étendant sur trois des six semestres de formation, l'institution place désormais la temporalité au cœur des processus. Les expériences de mobilité sont elles-mêmes pensées en termes de processus c'est-à-dire de «séquences d'action/interaction qui évoluent et dont les changements peuvent être associés aux changements des conditions structurelles» (Strauss \& Corbin, 2004, p. 201). Parler de processus, c'est donc postuler une série de phénomènes, chronologiques ou non, mais aboutissant à un résultat. Développer un regard anthropologique est forcément processuel (Berger, 2006). 


\section{L'amnésie des apprentissages}

Nous avons par ailleurs observé chez nos informateurs une forme d'amnésie, processus non seulement d'oubli de leurs apprentissages socio anthropologiques, linguistiques, interculturels au niveau professionnel et de leurs propres efforts mais surtout processus de naturalisation de leur rapport au français. Ces processus d'amnésie ne sont pas sans rappeler les effets de lissage dans la mise en récits, cette illusion biographique (Bourdieu, 1986) adaptée par Porcher (1987) au domaine des langues.

Il semble difficile pour nos informateurs de situer le dispositif dans leur parcours de formation et de juger des effets de celui-ci à un niveau professionnel. Ainsi c'était «juste intéressant» pour Patricia et surtout "c'était l'occasion de parler encore une fois, sur l'échange, mais maintenant c'est passé, alors, ça m'aide pas vraiment». (entretien avec Patricia) L'accent est mis sur la langue, sur l'opportunité d'utiliser de nouveau le français alors même que lors des deux phases que sont l'accompagnement puis le réinvestissement des expériences de mobilité, le français n'est pas forcément la langue véhiculaire. Patricia avait toutefois tenu à s'exprimer, elle, volontairement en français dans ce cadre. Pour elle, les réinvestissements de la mobilité se situent non pas dans la construction d'un habitus professionnel mais au niveau des compétences en langues: «On peut valoriser les connaissances en français, les connaissances de la langue» (entretien avec Patricia). Il y a un véritable décalage entre ce que souhaite faire travailler le dispositif (concepts convoqués, activités mises en place, etc.) et ce que prétendent retenir les acteurs au final, et ce, malgré le fait que ce dispositif soit le fruit d'une analyse préalable de leurs besoins et ait été conçu sur mesure pour leur terrain de formation. Cela fait écho aux observations faites par Reinhardt et Rosen (2013b, p. 81) avec un dispositif analogue utilisé pour encadrer les mobilités de médecins en formation initiale, les attentes des étudiants (beaucoup en langue et peu en sociolinguistique) se distinguent volontiers de celles des enseignants (qui au contraire attendent peu en langue et beaucoup en sociolinguistique). Peter ajoute d'ailleurs en entretien au sujet du dispositif: «Personnellement, à mon avis, on n'a pas besoin de ça».

\section{Travailler au-delà du temps de la formation}

Nous savons d'expérience que les trois années de formation initiale au métier de l'enseignement au niveau primaire à la PHBern sont intenses. Il y a souvent surcharge cognitive: trop et trop vite. Lorsqu'ils ne sont pas en stage dans les écoles, les étudiants sont en cours au sein de l'institution; pour qui veut l'effectuer en trois années, la formation est à temps plein et ne laisse pas ou peu de place à d'autres occupations professionnelles en parallèle. Avec 5 à 8 ECTS au total seulement, le FLE n'est qu'une matière parmi tant d'autres. Par ailleurs, au moment où l'on exige d'eux des réflexions métacognitives post expériences de mobilité, les acteurs sont déjà immergés dans la réalité de leur stage pratique suivant (en Suisse alémanique). Ils n'ont pas toujours «le temps de la réflexion» 
ni de la maturation nécessaire à la digestion de toutes les expériences de leur formation. On exige d'eux beaucoup, vite et dans des domaines très divers. Ainsi les objectifs processuels de formation semblent disproportionnés aux vues du temps de formation imparti. Dans le cas du FLE, il semble y avoir trop peu de reconnaissance (sous forme d'ECTS par exemple) et d'investissement en temps de formation à disposition pour développer les compétences nécessaires à un positionnement professionnel envers le contexte sociolinguistique présenté.

De plus, une durée de trois semestres de formation (soit un an et demi) est sans doute trop courte pour juger déjà d'un éventuel impact sur le rapport au français. Les processus réflexifs sont à peine enclenchés (quand ils le sont) et le temps de formation nous semble à la fois trop chargé et trop soudain pour mesurer les effets des processus ainsi enclenchés. Les exigences institutionnelles de réflexion arriveraient-elles trop tôt? Selon les acteurs, elles arriveraient pourtant trop tard ${ }^{15}$. Entre trop tôt et trop tard, les réflexions ne semblent jamais tomber à point. À ce stade, nous envisageons de travailler au-delà du temps de la formation initiale et de reprendre contact avec nos informateurs deux années après la fin de leur formation initiale. Ainsi, nous pensons compléter notre évaluation pilote du dispositif au printemps 2019.

\section{Conclusion}

À première vue, nous observons des évolutions au niveau des représentations du français mais aussi des décalages importants entre la conceptualisation de notre dispositif et sa mise à l'épreuve du terrain. Nous aimerions rappeler une fois de plus que, dans le cadre d'un travail thématisant le rapport au français sur un terrain aussi complexe et chargé de tensions sociolinguistiques, le champ d'action institutionnelle est limité par les représentations sociales largement partagées et énonçons nos objectifs scientifiques en toute humilité. Notre rôle éthique n'est-il pas de semer des graines et de tenter, encore et toujours, d'outiller les futurs enseignants de clés de compréhension du monde dans lequel ils évoluent et en particulier de l'environnement linguistique suisse (et de la proximité immédiate des langues à Berne)? C'est là ce que nous faisons dorénavant à l'aide des approches socio-anthropologiques en didactique des langues et des cultures. 


\section{Notes}

1 Le terme de communauté linguistique est issu de la Constitution de 1999 qui le définit dans le contexte suisse comme population ou groupe d'individus partageant de fait la même langue (p. 20).

2 Le Röstigraben, c'est littéralement «le fossé du rösti». Le rösti est un plat à base de pommes de terre et de fromage, dont on attribue l'origine à la Suisse alémanique. Le plat de pommes de terre est ici emblématique de tout ce qui oppose les Alémaniques aux Romands, à commencer par les pratiques culinaires donc, mais aussi les pratiques linguistiques, politiques, sociales, économiques, comportementales, etc. Le Röstigraben est une notion extrêmement présente dans les discours et les médias suisses (Brohy, 1997).

3 La PHBern dispose également d'autres instituts de formation initiale pour les futurs enseignants du secondaire (équivalents du collège et du lycée) et de l'enseignement spécialisé.

4 European Credits Transfer System. 1 ECTS correspond à environ trente heures de travail.

5 Soit Bac+3 en France.

6 Tous les prénoms sont fictifs.

7 Avec l'allemand et les mathématiques, le français constitue la $3^{\mathrm{e}}$ matière de sélection qui permet de poursuivre sa scolarité en voie générale au secondaire 1, puis d'intégrer le gymnase après la fin de la scolarité obligatoire. Un échec en français peut donc mener à une orientation professionnelle non souhaitée.

8 «Une recherche-action-formation (RAF) est une modalité de formation d'enseignants qui peut permettre de modifier en profondeur certaines représentations sociolinguistiques qui constituent les résistances idéologiques, et donc des obstacles, au développement de pratiques éducatives inclusives. [...] On y discute des représentations sociales des pratiques langagières, ce qui amène à expliciter les normes sociales et les jeux de pouvoir liés aux pratiques langagières». (Clerc, Richerme-Manchet, 2016, pp. 19-21)

9 Le choix de la cartographie permet une flexibilité graphique et créatrice dans l'épaisseur: les cartes peuvent être chronologiques, symboliques, etc. L'instrument permet aux acteurs de donner sens à leurs propres expériences et de les articuler entre elles (Robin, 2014).

10 Certains formateurs de la PHBern sont sur le mode du tutoiement avec leurs étudiants, c'était notre cas. Le tutoiement ne traduit ici aucune familiarité particulière avec l'étudiante.

11 Il s'agit de nouveau d'une traduction personnelle du document officiel, disponible en ligne et rédigé en allemand (Pädagogische Hochschule Bern, 2017).

12 Actualité du projet intitulé «Didactique de la mobilité» (Pädagogische Hochschule Bern, 2017).

13 Il ne s'agit aucunement d'une indication de genre. Nous avons conservé la langue - parfois incorrecte - des informateurs qui se sont ici exprimés en langue " étrangère ». Lisa voulait dire des «Français».

14 Voir le site du projet (Universität Bern, 2017).

15 Entre le moment du stage (juin) et les modules de réflexion (début octobre) les étudiants ont déjà eu un autre stage, obligatoire, dans une école alémanique en septembre; ils ont dès lors du mal à revenir alors sur ce qu'ils ne voient plus que comme leur avant-dernier stage. 


\section{Bibliographie}

Abric, J.-C. (1989/1997). Étude expérimentale des représentations sociales. In D. Jodelet (Ed.), Les représentations sociales (pp. 187-203). Paris: PUF.

Albero, B. (2010). La formation en tant que dispositif: du terme au concept. In B. Charlier \& F. Henri (Éd.), Apprendre avec les technologies (pp. 47-50). Paris: Presses Universitaires de France.

Anquetil, M. (2006). Mobilité Erasmus et communication interculturelle. Une recherche-action pour un parcours de formation. Berne: Transversales/ Peter Lang.

Auger, N., Molinié, M., Goï, C. \& Guillaumin, C. (2011). Analyse de dispositifs universitaires FLE/S d'accompagnement au développement de compétences réflexives. In A. Bretegnier (Éd.), Formation linguistique en contextes d'insertion. Compétences, posture, professionnalité: concevoir un cadre de référence(s) (pp. 177-194). Berne: Peter Lang,

Baudouin, J.-M. (2009). L'autobiographie à l'épreuve du texte. In D. Bachelart \& G. Pineau (Éd.), Le biographique, la réflexivité et les temporalités. Articuler langues, cultures et formation (pp. 97-109). Paris: L'Harmattan.

Berger, C. (2006). Démarche anthropologique et entretien compréhensif: approcher la réalité de l'Autre. In A. Cain \& G. Zarate (Ëd.), L'entretien: ses apports à la didactique des langues (pp. 17-27). Paris: Le Manuscrit.

Beuscart, J.-S. \& Peerbaye, A. (2006). Histoires de dispositifs. Terrains et travaux, 2, (11), 3-15.

Bourdieu, P. (1986). L'illusion biographique. Actes de la Recherche en Sciences Sociales ARSS, 62-63, 69-72.

Brohy, C. (1997). Prendre les images au mot: caricatures de presse évoquant le plurilinguisme en Suisse. In M. Matthey (Éd.), Les langues et leurs images (pp. 308-315). Le Mont-sur-Lausanne: LEP.

Calvet, L.-J. (1987). La guerre des langues et les politiques linguistiques. Paris: Éditions Payot.

Carroll, R. (1987). Les évidences invisibles. Paris: Éditions du Seuil.

Chopin, M.-P. (2010). Les usages du temps dans les recherches sur l'enseignement. Revue française de pédagogie, 170, 87-110.

Clerc, S. \& Richerme-Manchet, C. (2016). Aspects glottopolitiques d'une recherche-action-formation visant des pratiques éducatives inclusives de la pluralité sociolinguistique en terrain scolaire francophone. In P. Blanchet (Éd.), Politiques linguistiques et enseignement-apprentissage du français: quelles perspectives pour la pluralité linguistique? (pp. 19-27). Louvain.

Constitution fédérale de Confédération suisse du 18 avril 1999, Consulté dans https://www. admin.ch/opc/fr/classified-compilation/19995395/201801010000/101.pdf

Dervin, F. (2007). Evaluer l'interculturel: problématiques et pistes de travail. In F. Dervin \& E. Suomela-Salmi (Éd.), Evaluer les compétences langagières et interculturelles dans l'enseignement supérieur (pp. 95-122). Turku, Publications du département d'études françaises.

Develotte, C. (2006). Le Journal d'étonnement. Aspects méthodologiques d'un journal visant à développer la compétence interculturelle, LIDIL, 34, 105-124.

Doise, W. (1986). Les représentations sociales: définition d'un concept. In W. Doise \& A. Palmonari (Éd.), Textes de base en psychologie, l'étude des représentations sociales (pp. 81-94). Neuchâtel: Delachaux et Niestlé.

Goffman, E. (1973). La présentation de soi. Paris: Éditions de Minuit.

Gohard-Radenkovic, A. (2009). Peut-on former à la différence? Peut-on "penser la différence» dans la mobilité? Les Cahiers de l'APLIUT, 28, 10-23.

Gohard-Radenkovic, A. \& Veillette, J. (2015). Nouveaux espaces dans de nouvelles logiques migratoires? Entre mobilités et immobilités des acteurs. Cahiers internationaux de sociolinguistique, 8, 19-46.

Gohard-Radenkovic, A. (2016). Don et dette ou les fonctions du contre-don dans la construction des relations sociales. MediAzioni, 20, 1-25. Consulté dans http://www. 
mediazioni.sitlec.unibo.it/images/stories/PDF_folder/document-pdf/20-2016/5\%20 gohard.pdf

Goï, C. \& Huver, E. (2011). La réflexivité comme compétence professionnelle en formation universitaire: une nécessité professionnelle ou une injonction (de) dans l'air du temps? In A. Bretegnier (Ed), Formation linguistique en contextes d'insertion. Compétences, posture, professionnalité: concevoir un cadre de référence(s) (pp. 195-211). Berne: Peter Lang.

Jodelet, D. (Éd.). (1989/1997). Les représentations sociales. Paris: PUF.

Laplantine, F. (1987). L'anthropologie. Paris: Éditions Seghers.

Morin, E. (1959). Autocritique. Paris: Points Essais.

Morin, E. (2005). Introduction à la pensée complexe. Paris: Éditions du Seuil.

Morlaix, S. (2006). Qualité du temps, efficacité de l'apprentissage. Administration et éducation, $111,88-89$.

Murphy-Lejeune, E. \& Zarate, G. (2003). L'acteur social pluriculturel: évolution politique, positions didactiques. Recherches et applications/ Le français dans le monde, Numéro spécial, $32-46$.

Pädagogische Hochschule Bern. (2017). Expériences de mobilité. Consulté dans https:// www.phbern.ch/studiengaenge/vp/studium/studienbereiche/fachwissenschaften-und-fachdidaktiken/experiences-de-mobilite.html

Pädagogische Hochschule Bern. (2017). Didactique de la mobilité. Consulté dans https:// www.phbern.ch/didactique-de-la-mobilite.html

Paillé, P. \& Mucchielli, A. (2012). L'analyse qualitative en sciences humaines et sociales. Paris: Armand Colin.

Pasquini, R. (2013). Quand les récits de pratique enseignante parlent d'apprentissage. Laval: Presses de l'Université de Laval.

Peeters, H. \& Charlier, P. (1999). Contributions à une théorie du dispositif, Hermès, 25, 15-23.

Porcher, L. (1987). Champs de signes: États de la diffusion du français langue étrangère. Paris: Crédif/ Didier.

Porcher, L. (1997). Lever de rideau. In G. Zarate (Éd.), Les représentations en didactique des langues et cultures. Paris: ENS de Fontenay/ Saint-Cloud.

Reinhardt, C. \& Rosen, E. (2013a). Le journal d'étonnement comme capital en contexte de mobilité européenne. In G. Alao, M. Derivry, E. Suzuki \& S. Yun-Roger (Éd.), Didactique plurilingue et pluriculturelle. L'acteur en contexte mondialisé (pp. 135-142). Paris: Editions des Archives Contemporaines.

Reinhardt, C. \& Rosen, E. (2013b). La place des éponymes dans une préparation à la mobilité étudiante. Journal of International Mobility, 1, 79-91.

Robin, J. (2013). Séjour de mobilité linguistique obligatoire dans la formation des enseignants: oui, mais comment? Le cas de la PHBern. Babylonia, 1, 94-98.

Robin, J. (2014) Cartes de langue(s) et de mobilité(s) de futurs enseignants du primaire à Berne: quand une dynamique dialogique entre les corpus dévoile des représentations du français. Glottopol, 24, 64-79.

Robin, J. (2015a,). "Ils aiment pas le français». Le rapport au français de futurs enseignants du primaire de la PHBern dans leurs récits de formation et de mobilité. Berne: Peter Lang.

Robin, J. (2015b). Un semestre Erasmus comme «interstice institutionnel» ou quand le séjour de mobilité imposé par la formation des enseignants du primaire engendre des formes d'immobilités, Cahiers internationaux de sociolinguistique, 8, 201-223.

Robin J. (2017a). Didactique de la mobilité: le travail des représentations dans la préparation, l'accompagnement et le réinvestissement des expériences. Le Langage et l'Homme, 1, 61-75.

Robin, J. (2017b). Expériences de mobilité: apports et limites des sciences sociales dans un dispositif institutionnel de formation en français langue étrangère. In E. Argaud \& al. (Éd.). Le proche et le lointain/ The near and the far: enseigner, apprendre et partager des cultures étrangères/Teaching, Learning and Sharing of Foreign Cultures (pp. 185-194). Paris: 
Editions des Archives Contemporaines.

Simmel, G. (1999). Excursus sur l'étranger. In Sociologie. Études sur les formes de la socialisation (pp.- 663-668). Paris: PUF.

Strauss, A. \& Corbin, J. (2004). Les fondements de la recherche qualitative. Techniques et procédures de développement de la théorie enracinée. Fribourg: Academic Press Fribourg.

Universität Bern. (2017). MILSA Mentoring Intercultural Learning Through Study Abroad. Consulté dans http://www.milsa.unibe.ch/

Veillette, J. \& Gohard-Radenkovic, A. (2016). Culture, cultures... et interculturel. In C. Blons-Pierre \& P. Banon (Éd.), Didactique du français langue étrangère et seconde dans une perspective plurilingue et pluriculturelle (pp. 203-227). Berne: Peter Lang.

Vinatier, I. (2009). Pour une didactique professionnelle de l'enseignement. Rennes: Presses Universitaires de Rennes.

Widmer, J. (2004). Langues nationales et identités collectives. Le cas de la Suisse. Paris: L'Harmattan.

Windisch, U. (2007). Le modèle suisse. Lausanne: L'Âge d'Homme.

Zarate, G. (1993). Représentations de l'étranger et didactique des langues. Paris: Didier.

Zarate, G. (1997). Les représentations en didactique des langues et cultures. Paris: Revue de l'ENS de Fontenay/ Saint-Cloud.

Zarate, G. (2012). L'expérience de la pluralité en situation de mobilité internationale dans la formation d'un capital plurilingue et pluriculturel. In O. Bertrand (Éd.), Diversités culturelles et apprentissage du français. Approche interculturelle et problématiques linguistiques (pp. 11-17). Paris: Éditions de l'École Polytechnique.

Mots-clés: Didactique des langues et des cultures, didactique de la mobilité, formation initiale des enseignants, dispositif institutionnel, représentations sociales

\section{Die Entwicklung von sozialen Repräsentationen des Französischen bei angehenden Berner Primarlehrpersonen - Welche Rolle spielt der Faktor Zeit in der Grundausbildung?}

\section{Zusammenfassung}

Das Institut Vorschulstufe und Primarstufe der Pädagogischen Hochschule Bern bildet in einem dreijährigen Bachelorprogramm deutschsprachige Primarlehrpersonen aus. Das Fach Französisch ist für alle Studierenden obligatorisch, ebenso wie ein Sprachaufenthalt im französischen Sprachraum. Ziel ist der Erwerb sprachlicher und interkultureller Kompetenzen in Verbindung mit professionellen Handlungskompetenzen. Basierend auf sozial-anthropologischen Ansätzen zielt das Programm auch auf die Bearbeitung der sozialen Repräsentationen des Französischen. Es umfasst eine Vorbereitung, die Unterstützung während des Aufenthalts und eine Reflexion im Anschluss an den Aufenthalt. Die Zwischenbilanz des Programms ist allerdings durchzogen. Eine Erkenntnis aus den Analysen ist, dass die Rolle des Faktors Zeit für die Entwicklung der Beziehung zum Französischen stärker als bisher berücksichtigt werden sollte.

Schlagworte: Didaktik der Sprachen und der Kulturen, Mobilitätsdidaktik, Grundausbildung Institutionelles Umfeld Repräsentationen 


\section{L'evoluzione delle rappresentazioni del francese dei futuri insegnanti svizzero-tedeschi in formazione iniziale: una questione legata ai tempi della formazione?}

\section{Riassunto}

In risposta ai bisogni identificati nelle nostre ricerche precedenti, la nuova formazione iniziale in francese lingua straniera proposta dalla Pädagogische Hochschule Bern agli insegnanti svizzero-tedeschi di scuola elementare si basa su approcci socio-antropologici. Un dispositivo sistemico di "didattica della mobilità" costruito in tre parti (preparazione, accompagnamento, reinvestimento/riflessione) articola le esperienze di mobilità nello spazio francofono con la pratica professionale, i bisogni linguistici e il lavoro sulle rappresentazioni sociali del francese. Gli effetti di questo dispositivo di formazione, concepito su misura per il pubblico in questione, presentano tuttavia dei limiti e ci portano a considerare i tempi della formazione e il loro ruolo nella costruzione del rapporto al francese. Parole-chiave:

Parole chiave: Didattica delle lingue e delle culture, didattica della mobilità, formazione iniziale degli insegnanti, dispositivo istituzionale, rappresentazioni sociali

\section{Evolution(s) of the représentations sociales on French language amongst German-speaking future teachers in Berne: a matter of educational time?}

\section{Abstract}

The Institut Vorschulstufe und Primarstufe at the Pädagogische Hochschule Bern (PHBern) trains future German-speaking primary school teachers with a three years Bachelor program. It is compulsory for all students to study French as a subject and to attend a professional mobility experience in a French-speaking environment so that they articulate linguistic and intercultural competences with professional competences. The new learning environment set to supervise mobility, including preparation (before), support (during) and reflection (after), is based on social anthropological approaches and addresses the notion of représentations sociales. Through the analysis of case studies, we will see how time influences the process at stake.

Keywords: Language learning and teaching, Mobility didactics, Teacher education, Institutional learning environment, French notion of représentations sociales 
